\title{
Microribonucleic Acid-15a-5p Alters Adriamycin Resistance in Breast Cancer Cells by Targeting Cell Division Cycle-Associated Protein 4
}

\author{
Jiang-Tao Zhang' \\ Jun Chen ${ }^{2}$ \\ Hui-Chao Ruan' \\ Feng-Xi Li ${ }^{3}$ \\ Sen Pang' \\ Yu-Ju Xu' \\ Dao-Lai Huang' \\ Xiang-Hua $\mathrm{Wu}^{\prime}$ \\ 'Department of Gastrointestinal and \\ Gland Surgery, First Affiliated Hospital of \\ Guangxi Medical University, Nanning, \\ Guangxi Zhuang Autonomous Region, \\ People's Republic of China; ${ }^{2}$ Department \\ of Thyroid and Breast Surgery, \\ Guangzhou Panyu Central Hospital, \\ Guangzhou, Guangdong Province, \\ People's Republic of China; ${ }^{3}$ Department \\ of Gastrointestinal Surgery, Guangxi \\ International Zhuang Medicine Hospital, \\ Nanning, Guangxi Zhuang Autonomous \\ Region, People's Republic of China
}

Correspondence: Xiang-Hua Wu Department of Gastrointestinal and Gland Surgery, First Affiliated Hospital of Guangxi Medical University, No. 6, Shuangyong Road, Qingxiu District, Nanning, 53002I, Guangxi Zhuang Autonomous Region, People's Republic of China

Tel +86 77I-535670I

Fax +86-77I-535003।

Email wuxianghuag@163.com
Objective: Although chemotherapy is one of the first line clinical treatment of tumors, the efficacy of chemotherapy has been severely restricted by the frequent occurrence of drug resistance phenomenon. Multiple studies found that miRNAs can regulate the chemosensitivity of tumor cells. Here, this study aimed to assess the potential role of the miR-15a-5p/ cell division cycle-related protein 4 (CDCA4) axis in breast cancer (BC) resistance to Adriamycin.

Methods: In the present study, the relative expression of miRNA-15a-5p in MCF-7/ADR, MCF-7 and Hs578Bst was measured by qRT-PCR. MCF-7/ADR cells underwent transfection with an miR-15a-5p mimic and inhibitor, respectively. Transwell assays, flow cytometry and CCK8 were performed to examine the potential effects of the abnormal expression of miR$15 a-5 p$. The association of aberrant miR-15a-5p expression with Adriamycin resistance in $\mathrm{BC}$ was determined in cultured MCF-7/ADR cells. Bioinformatics was employed to predict the genes targeted by miR-15a-5p. Moreover, the correlation between miR-15a-5p and its target gene, CDCA4, was evaluated based on qRT-PCR data.

Results: The expression of miR-15a-5p was significantly downregulated in MCF/ADR cells compared with MCF-7 and Hs578Bst cell lines. In the presence of Adriamycin, miR-15a-5p overexpression significantly increased cell chemosensitivity, as well as MCF-7/ADR cell proliferation, invasion, and migration, while promoting apoptosis and inducing cell-cycle arrest in the synthesis phase. CDCA4 RNA interference enhanced these effects as shown in our previous study. Bioinformatics identified CDCA4 as an miR-15a-5p target gene. qRTPCR further demonstrated that CDCA4 and miR-15a-5p expression levels were inversely correlated.

Conclusion: Adriamycin resistance in BC cells was, at least in part, altered by mRNA-15a$5 \mathrm{p}$ via regulation of its target gene, $\mathrm{CDCA} 4$, by controlling the cell cycle, which may provide some novel ideas for BC chemotherapy in the future.

Keywords: breast cancer, Adriamycin resistance, miR-15a-5p, CDCA4, cell-free nucleic acids, molecular targets, breast cancer patient stratification

\section{Background}

\section{Resistance to Adriamycin Treatment in Breast Cancer}

Breast cancer (BC) represents one of the commonest malignant tumors among females. The second most fatal tumor type after lung cancer, BC seriously affects many women physically and mentally. ${ }^{1}$ Breast cancer is considered a systemic disease. With an increased understanding of BC's biological features and the use 
of currently modified and updated therapeutic approaches, BC treatment has been developed as a comprehensive therapy that includes surgical, radiotherapeutic, chemotherapeutic, and endocrine treatment procedures, as well as biological targeting and other treatment methods. Chemotherapy is a crucial BC treatment and one in which Adriamycin is widely used. Studies have described chemotherapy resistance as one of the most critical causes of cancer treatment failure, and Adriamycin resistance frequently promotes BC progression and recurrence. However, the mechanism by which Adriamycin resistance occurs in $\mathrm{BC}$ remains unclear. Therefore, $\mathrm{BC}$ resistance to Adriamycin treatment must be urgently investigated.

\section{miRNAs and Treatment Resistance in Tumors}

Microribonucleic acids (miRNAs) are short non-coding RNAs with lengths of approximately 20-24 nucleotides that regulate genes at the post-transcriptional level by binding specific parts of the three prime untranslated region (3'-UTR) of the target gene and affect both translation and stability. ${ }^{2}$ At present, the miRNA database (miRBase) includes more than 2500 different miRNAs that are associated with cell proliferation, invasion, metastasis, and apoptosis in a variety of tumors. Increasing evidence suggests that miRNAs play a critical role in $\mathrm{BC}$ occurrence and progression. ${ }^{3-5}$ Cell free nucleic acid (CFNAs) refers to the release of free DNA, free RNA and mitochondrial DNA into the human blood circulation because of cells necrosis and apoptosis. Compared with tissue biopsy, liquid biopsy has the characteristics of minimal trauma and strong heterogeneity. The miRNAs in the blood circulation have been found to hold great potential for early cancer detection and monitoring. In BC, miR-21, miR-182 and miR-451 in blood have been illustrated to have a close link with tumor size and lymphatic metastasis. ${ }^{6}$ Moreover, miRNAs are also significantly associated with treatment resistance in tumors. Existing findings confirmed that altered miR-222 mediates BC resistance to Adriamycin via regulation of Forkhead box O1 (FOXO1). ${ }^{7}$ Additional data demonstrated that miR3609 sensitizes BC to Adriamycin by regulating the expression of programmed death-ligand $1 .{ }^{8}$ Recent research indicated that dysregulated miR-122-5p was closely related to Adriamycin resistance in BC. ${ }^{9}$ Several studies have indicated that disordered miRNAs were significantly associated with Adriamycin resistance in BC.

\section{miR-I5a and Tumors}

Increasing evidence indicates that miR-15a is associated with the occurrence and development of a variety of malignant tumors, eg, cholangiocarcinoma, ${ }^{10}$ colon cancer, ${ }^{11}$ lung cancer, ${ }^{12}$ and BC. ${ }^{13}$ These studies revealed that miR-15a plays a critical role in multiple cancers. Fesler et al demonstrated that miR-15a can improve the therapeutic outcomes of patients with advanced bowel cancer by inhibiting the expression of many crucial genes, such as: B cell lymphoma 2 (BCL-2), B lymphoma Mo-MLV insertion region 1 (BMI-1), yesassociated protein 1 (YAP1) and doublecortin-like kinase 1 (DCLK), and may thus constitute a novel potential therapeutic target. The same study also demonstrated that reduced miR-15a amounts were associated with poor patient prognosis in colon cancer. ${ }^{14}$ However, the relationship between miR-15a and Adriamycin resistance in $\mathrm{BC}$, and the underlying molecular mechanisms involved therein, remain largely undefined.

\section{CDCA4 and Resistance to Adriamycin in Breast Cancer}

Cell division cycle-associated protein 4 (CDCA4) belongs to the family of selected INK4A (SEI) genes. Several studies confirmed the overexpression of SEI-family genes to be closely related to tumorigenesis. ${ }^{15,16}$ The mRNA expression of CDCA4 determines the fate of cells by regulating the JUN proto-oncogene at the mRNA level. ${ }^{17}$ Research revealed that CDCA4 upregulation promoted cell entry into the first growth/synthesis (G1/S) phase; indeed, CDCA4 plays an important role in cell-cycle regulation by controlling activity of E2F transcription factors. ${ }^{18}$ The low expression of CDCA4 inhibits BC MDA-MB-231 cells. ${ }^{19}$ Moreover, we previously demonstrated that CDCA4 knockdown suppressed the proliferation and induced apoptosis in Adriamycin-resistant Michigan Cancer Foundation-7/ breast cancer cell line (MCF-7/ADR). ${ }^{20}$

\section{The Relationship of CDCA4 and miR-I5a-5p}

Alderman et al demonstrated that CDCA4 was a direct target of miRNA-15a via the luciferase reporter assay; the study also found that miRNA-15a could inhibit the growth and invasiveness of melanoma cells by binding to the 
3'UTR of CDCA4. ${ }^{21}$ Nevertheless, the role and relationship of miR-15a-5p with its target gene (CDCA4) in Adriamycin-resistant $\mathrm{BC}$ remains undefined. In the current study, miR-15a-5p levels were examined in different cell lines using the quantitative reverse transcriptionpolymerase chain reaction (qRT-PCR) method. The results showed that, compared with human mammary gland cells (Hs578Bst), MCF-7/ADR and MCF-7 cells had reduced miR-15a-5p amounts, particularly MCF-7/ADR cells. Additionally, high miR-15a-5p amounts could entirely or partially blunt Adriamycin resistance in BC cells, suppressing proliferation, invasiveness, and migration and induce apoptosis in MCF-7/ADR cells exposed to Adriamycin. We previously demonstrated that CDCA4 knockdown suppressed the proliferation of MCF-7/ADR cells and induced apoptosis in MCF-7/ADR cells administered with Adriamycin. These findings corroborated the data obtained following miR-15a-5p overexpression in the present study.

\section{Materials and Methods Cells}

Breast cancer MCF-7/ADR, MCF-7, and Hs578Bst cells were provided by the Type Culture Collection of the Chinese Academy of Sciences (China). The MCF-7/ADR and parental MCF-7 cell lines were cultured in an RPMI1640 medium (Gibco, USA), and Hs578Bst cells were cultured in Dulbecco's modified eagle medium (DMEM) (Gibco). In the present study, both DMEM and RPMI1640 included 10\% fetal bovine serum (FBS; Gibco), as well as streptomycin $(100 \mathrm{mg} / \mathrm{mL})$ and penicillin $(100 \mathrm{U} /$ $\mathrm{mL}$ ). To preserve Adriamycin resistance in MCF-7/ADR cells, a cell culture procedure was performed with $1 \mu \mathrm{g} /$ $\mathrm{mL}$ Adriamycin (Selleck, USA) in a humid environment with $5 \%$ carbon dioxide $\left(\mathrm{CO}_{2}\right)$ at $37^{\circ} \mathrm{C}$.

\section{Real-Time Polymerase Chain Reaction}

Total RNA extraction from cells was carried out using a TRIzol reagent (Shanghai Pufei Biotechnology, China) following the manufacturer's instructions, and complementary deoxyribonucleic acid (cDNA) synthesis was conducted using a first-strand cDNA synthesis kit (TaKaRa, Japan). Specific reverse transcription primers were designed by RiboBio (China). A SYBR Premix Ex Taq (TaKaRa, Japan) instrument was used to conduct the qRT-PCR, which was performed according to the following reaction conditions: pre-denaturation at $95^{\circ} \mathrm{C}$ for $30 \mathrm{~s}$; amplification in 45 cycles of $95^{\circ} \mathrm{C}(5 \mathrm{~s}), 60^{\circ} \mathrm{C}(30 \mathrm{~s})$, and $72^{\circ} \mathrm{C}(3 \mathrm{~s})$. The delta-delta $\mathrm{Ct}$ method was used to conduct data analysis. The following primer sequences were used in the qRT-PCR analysis: miR-15a-5p reverse, 5'-ACG TAG CAG CAC ATA ATG GTT TGTG-3'; U6 reverse, 5'-GAA GAT TAG CAT GGC CCC TGC-3' GCA; miR-15a-5p mimics, forward 5'-UAG CAG CAC AUA AUG GUU UGUG-3', and reverse 5'-CAA ACC AUU AUG UGC UAUU-3'; miR15a-5p inhibitor, forward 5'-CAC AAA CCA UUA UGU GCU-3'; CDCA4, forward 5'-ATT TGA AAC GCT GGA GACT-3', and reverse 5'-CCC ATC ATG CCT GTC AGTA3'; glyceraldehyde-3-phosphate dehydrogenase, forward 5'TGA CTT CAA CAG CGA CAC CCA-3', and reverse 5'CAC CCT GTT GCT GTA GCC AAA-3'. qRT-PCR was repeated by three times.

\section{Cell Transfection}

The miR-15a-5p mimic/inhibitor and their respective negative controls were synthesized by RiboBio (China). The MCF-7/ADR cells were seeded in 24-well plates at 1 x $10^{5} /$ well. Transfection was performed at $40 \%$ confluence with the miR-15a-5p mimic (50 nM) or inhibitor (100 nM) as directed by the manufacturer. These cells were assessed in subsequent experiments after $48 \mathrm{~h}$ of incubation. Then, qRT-PCR was carried out for miR-15a-5p detection in MCF-7/ADR cells upon transfection.

\section{Cell Counting Kit-8}

Cell counting kit-8 (CCK-8) was carried out for detecting cell proliferation at various Adriamycin concentrations. Upon transfection (the miR-15a-5p mimic or inhibitor, or respective negative controls), $\mathrm{MCF}-7 / \mathrm{ADR}$ cells were seeded into 96 -well plates at $2 \times 10^{3} /$ well. Following overnight culture, the cells were administered with five distinct levels of Adriamycin $(2.5,5,10,20$, and $40 \mu \mathrm{g} / \mathrm{mL}$, respectively) in quintuplicate and incubated for $48 \mathrm{~h}$. Then, $10 \mu \mathrm{L}$ of CCK-8 reagent (Dojindo, Japan) was added per well and incubated for $2 \mathrm{~h}$. Subsequently, optical density at $450 \mathrm{~nm}$ was obtained on a microplate reader. The half-maximal inhibitory concentration (IC50) of the control group was used as the Adriamycin concentration in subsequent experiments. The inhibition rate was derived as follows: inhibition rate $=($ ODtest-ODblank $) /($ ODcontrol-ODblank $) \times 100 \%$. The experiments were performed in triplicate.

\section{Transwell Assays}

Transwell assays were performed to measure the invasive and migratory abilities of cells. The miR-15a-5p mimic, miR-15a-5p inhibitor, and control groups were cultured 
with Adriamycin (6 $\mu \mathrm{g} / \mathrm{mL}$ as the IC50 of the control group) before conducting the experiment. Next, cells at $2 \times 10^{4} / \mathrm{mL}$ in a culture medium without serum were seeded into the upper chambers of the transwell plates, while the lower chambers received $700 \mu \mathrm{L}$ medium containing 20\% FBS. Following the cell culture at $37^{\circ} \mathrm{C}$ in the presence of $5 \%$ $\mathrm{CO}_{2}$ for $16 \mathrm{~h}$, the cells that had migrated into the lower chambers underwent $4 \%$ paraformaldehyde fixation for 20 min. Then, non-migrated cells were wiped, and crystal violet staining was performed for $20 \mathrm{~min}$. The migrated cells were counted using the ImageJ (V 1.8.0) software program in three randomly selected fields of view. In the cell invasion assay, the upper chambers of the transwell plates underwent Matrigel (BD Biosciences, USA) pre-coating and were maintained at $37^{\circ} \mathrm{C}$ for $1 \mathrm{~h}$. Then, the cells were seeded into the upper chambers in a serum-free medium. The remaining experimental steps were as described above for the cell migration assay. Triplicate assays were carried out.

\section{Flow Cytometry}

Apoptosis and cell-cycle distribution were assessed using flow cytometry. After incubation with Adriamycin $(6 \mu \mathrm{g}$ / $\mathrm{mL}$ ) for $24 \mathrm{~h}$, the cells were harvested with ethylenediaminetetraacetic acid (EDTA)-free trypsin and washed twice with a D-Hanks solution. Then, a $5 \mu \mathrm{L}$ Annexin V-APC assay was added for $15 \mathrm{~min}$ at an ambient temperature and shielded from light. Data analysis was carried out using a fluorescence-activated cell sorting (FACS) analyzer (BD Biosciences, USA).

Following the same procedure described for the cellular apoptosis assessment, the cells were washed and a permeabilization $(10 \mu \mathrm{L})$ and propidium iodide (PI) staining solution $(5 \mu \mathrm{L})$ were added. Following incubation for $30 \mathrm{~min}$ away from light and at an ambient temperature, the cell-cycle distribution was analyzed using the FACS analyzer. The experiments were performed in triplicate.

\section{Statistical Analysis}

Data were derived as the mean \pm standard deviation and assessed using the SPSS Statistics 23.0 software program. The Student's $t$-test was performed to compare groups; $\mathrm{P}<$ 0.05 indicated a statistical significance.

\section{Results}

\section{The Expression Levels of}

\section{Microribonucleic Acid-I 5a-5p in Different Cell Lines}

The MCF-7/ADR, MCF-7, and Hs578st cells were comparatively assessed by qRT-PCR for the expression of miR-15a-5p. As shown in Figure 1, miR-15a-5p was markedly downregulated in MCF-7/ADR and MCF-7 cells compared with Hs578st cells. In addition, compared with MCF-7 cells, MCF-7/ADR cells showed more pronounced miR15a-5p downregulation (Figure 1A; $\mathrm{P}<0.05$ ). Next, MCF7/ADR cells underwent transfection with an miR-15a-5p mimic or inhibitor, and qRT-PCR was carried out to detect miR-15a-5p amounts in transfected and control MCF-7/ ADR cells. In this study, miR-15a-5p amounts were dramatically decreased and increased after transfection with the miR-15a-5p inhibitor and mimic, respectively, compared with the control cells (Figure 1B; $\mathrm{P}<0.05$ ).

\section{A}

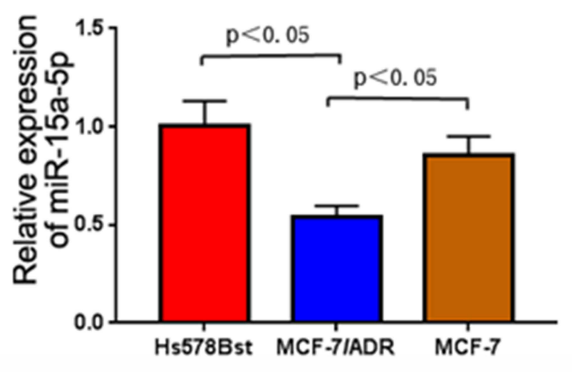

B

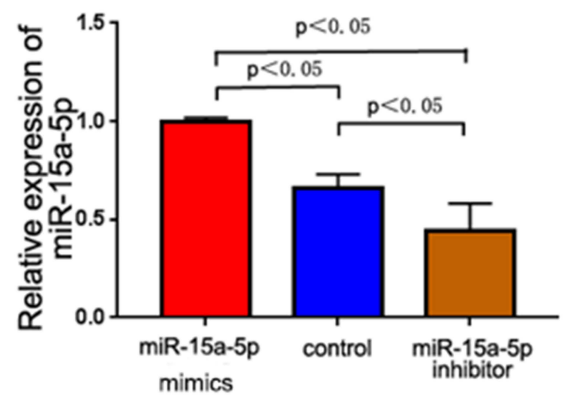

Figure I The relative expression of microribonucleic acid-15a-5p (miR-15a-5p) in breast cancer cells. (A) The relative expression of miR-I5a-5p in normal diploid myoepithelial, Michigan Cancer Foundation-7 (MCF-7) and Adriamycin (ADR)-resistant breast cancer cells MCF-7 (MCF-7/ADR); miR-I5a-5p expression was significantly decreased in MCF-7/ADR cells $(P<0.05)$. (B) The relative expression of miR-15a-5p in non-transfected MCF-7/ADR cells and those transfected with an miR-I5a-5p mimic and inhibitor, respectively. The MiR-I5a-5p amounts were dramatically decreased and increased after transfection with an miR-I5a-5p inhibitor and mimic, respectively, compared with control cells $(P<0.05)$. 


\section{Differentially Expressed Microribonucleic Acid-15a-5p Regulates Breast Cancer Cell Resistance to Adriamycin}

Cell counting kit-8 was performed to investigate the cell viability of MCF-7/ADR and to determine the resistance of MCF-7/ADR and transfected MCF-7/ADR cells to Adriamycin. The results showed that miR-15a-5p overexpression in the miR-15a-5p mimic group significantly increased cell viability at various Adriamycin levels compared with the miR-15a-5p inhibitor and control groups. Conversely, miR-15a-5p downregulation in the miR-15a-5p inhibitor group dramatically reduced cell viability (Figure 2; $\mathrm{P}<0.05)$. The IC50 of Adriamycin upon transfection with the miR-15a-5p mimic was $1.934 \pm 0.252 \mu \mathrm{g} / \mathrm{mL}$, while $11.800 \pm$ $0.219 \mu \mathrm{g} / \mathrm{mL}$ and $6.517 \pm 0.166 \mu \mathrm{g} / \mathrm{mL}$ were obtained in the miR-15a-5p inhibitor and control groups, respectively.

\section{Differentially Expressed Microribonucleic} Acid- I5a-5p is Strongly Associated with Cell Invasion, Migration, and Apoptosis in Adriamycin-Resistant Breast Cancer Cells

The migratory and invasive abilities of cells were detected using transwell assays. Compared with the control group, miR-15a-5p overexpression (the miR-15a-5p mimic group) reduced the invasive and migratory abilities of MCF-7/ADR cells in the presence of Adriamycin. Conversely, miR-15a-5p downregulation resulted in the

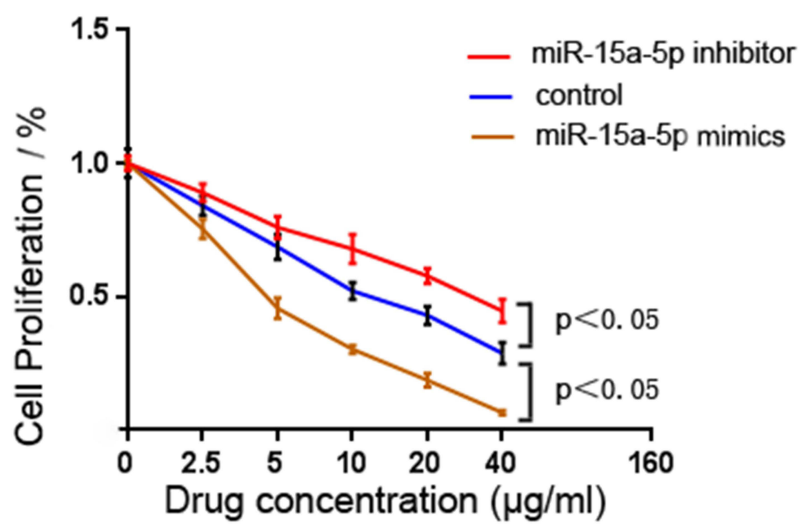

Figure 2 The cell viability of non-transfected Adriamycin (ADR)-resistant breast cancer cells MCF-7 (MCF-7/ADR) and those transfected with a microribonucleic acid-15a-5p (miR-15a-5p) mimic and inhibitor. Cell viability was reviewed by conducting cell counting kit-8. The overexpression of miR-15a-5p in the mimic group significantly increased the cell viability at various Adriamycin levels, compared with the miR-15a-5p inhibitor and control groups $(P<0.05)$. Conversely, miR-15a-5p downregulation in the inhibitor group dramatically reduced the cell viability $(P<0.05)$ opposite outcome in the miR-15a-5p inhibitor group (Figure 3) $(\mathrm{P}<0.05)$. The above data revealed that aberrant miR-15a-5p expression showed a significant association with $\mathrm{BC}$ cell invasion and migration, particularly under Adriamycin pressure.

Apoptosis and cell-cycle distribution in MCF-7/ADR cells were assessed using flow cytometry. The results showed that apoptosis had been induced to a significant degree in all three groups after treatment with Adriamycin. However, compared with the control group, the miR-15a$5 \mathrm{p}$ mimic and inhibitor groups showed remarkably increased and decreased apoptotic rates, respectively (Figure 4A) $(\mathrm{P}<0.05)$. The above findings suggested that miR-15a-5p overexpression in MCF-7/ADR cells significantly promoted apoptosis, particularly under Adriamycin pressure. Conversely, under the same conditions, low miR-15a-5p expression inhibited MCF-7/ADR cell apoptosis. Accordingly, these findings suggested that miR-15a-5p overexpression enhanced BC cell sensitivity to Adriamycin and led to decreased MCF-7/ADR cell invasion and migration, as well as induced apoptosis, in the presence of Adriamycin.

Cell-cycle distribution was also assessed using flow cytometry. Proportions of the S-phase cells were increased in all three MCF-7/ADR cell groups upon Adriamycin administration, particularly in the miR-15a-5p mimic group.

The rates of cells that were arrested in the S-phase were significantly elevated and reduced in the miR-15a$5 \mathrm{p}$ mimic and inhibitor groups, respectively, compared with the control cells. Furthermore, there were no marked differences in G1 and G2-phase cells, with or without Adriamycin administration $(\mathrm{P}<0.05)$ (Figure 4B). These findings revealed that miR-15a-5p overexpression blunted MCF-7/ADR cell proliferation through cell-cycle alteration, and may suppress MCF-7/ADR cell resistance to Adriamycin.

\section{Cycle-Associated Protein 4 is a Direct Target Gene of Microribonucleic Acid-I 5a-5p}

Next, we aimed to assess the association of CDCA4 with miR-15a-5p and to determine whether these molecules were involved in Adriamycin resistance in BC. Potential miR-15a-5p targets were assessed using bioinformatics, based on information acquired from the miRDB, TargetScanHuman and PicTar databases. The data 


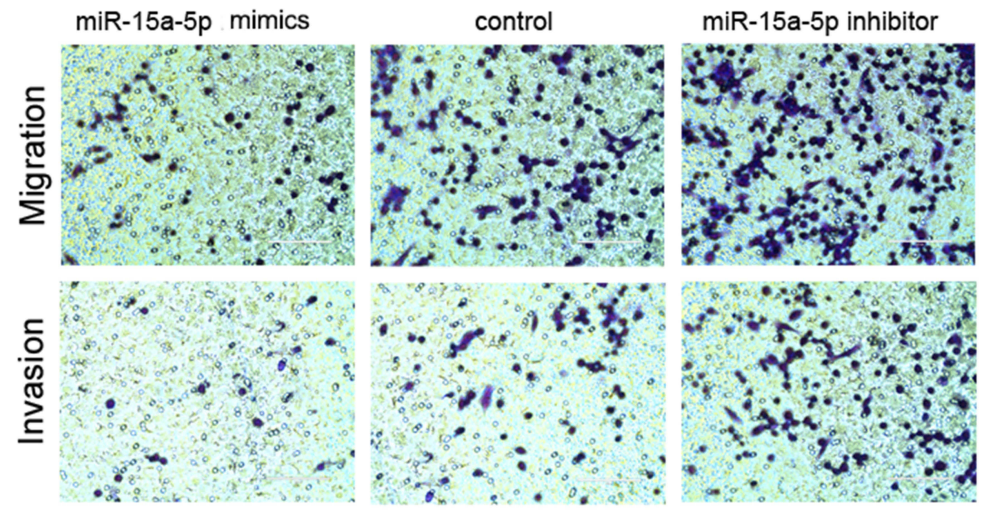

A

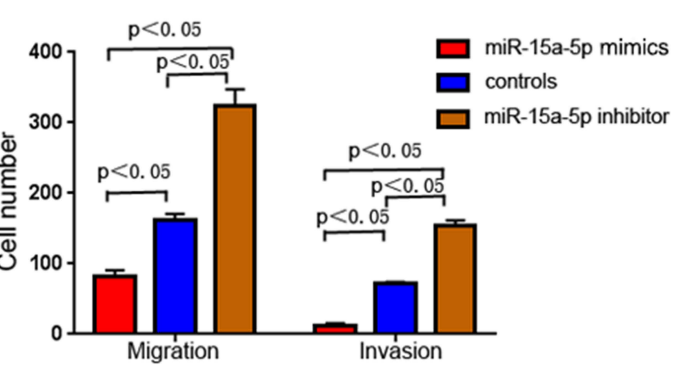

B

Figure 3 The migratory and invasive abilities of cells as detected by transwell assays. (A, B) Compared with the control group, microribonucleic acid- I5a-5p (miR-I5a-5p) overexpression (miR-15a-5p mimic group) inhibited invasion and migration of Adriamycin (ADR)-resistant breast cancer cells MCF-7 (MCF-7/ADR). Conversely, miR-I5a-5p downregulation resulted in the opposite outcomes for the miR-15a-5p inhibitor group $(P<0.05)$.

retrieved from the three online databases, as well as a luciferase reporter assay (as per Alderman et $\mathrm{al}^{20}$ ), identified the CDCA4 gene as being an miR-15a-5p target (Figure 5A). To further confirm that miR-15a-5p targeted CDCA4, CDCA4 mRNA amounts were evaluated by qRTPCR in transfected and control MCF-7/ADR cells. Compared with control mRNA levels, CDCA4 was upregulated and downregulated in the miR-15a-5p inhibitor and mimic groups, respectively $(\mathrm{P}<0.05)$ (Figure 5B). These findings indicated a negative correlation between CDCA4 amounts and miR-15a-5p levels in MCF-7/ADR cells. Additionally, we previously demonstrated that CDCA4 expression was associated with MCF-7/ADR cell viability, with low CDCA4 expression inhibiting cell proliferation and inducing apoptosis with or without Adriamycin administration. The above data indicated that low CDCA4 expression mimicked miR-15a-5p overexpression. Therefore, miR-15a-5p overexpression could alter MCF-7/ADR cell resistance to Adriamycin, inhibiting cell viability and inducing apoptosis via the regulation of CDCA4.

\section{Discussion}

\section{The Limitation of Treatment of Breast Cancer}

Breast cancer represents a universal health challenge for women. With the development of new approaches and treatment options for $\mathrm{BC}$, patient prognosis has somewhat improved. Chemotherapy has an increasingly crucial function in the comprehensive treatment of BC. Adriamycin is the primary chemotherapeutic agent in standard chemotherapy approaches for treating $\mathrm{BC}$ but resistance significantly limits its therapeutic effect and can result in treatment failure. ${ }^{22}$ The mechanism of Adriamycin resistance in $\mathrm{BC}$ remains unclear and requires further investigation.

\section{The Role of miRNAs in Breast Cancer}

Due to most tumors have metastasized far away when diagnosed, how to find early tumors have been focuses of research. Studies showed that state of chronic inflammation can reflect the occurrence and development of cancers. Chronic inflammation can lead to DNA injury, detection of abnormal cfDNA, miRNAs and methylation pattern in body fluid, which assists in diagnosis and grading of cancers. In summary, Cell-free nucleic is a promising biomarker, which is used for diagnosis, prognosis and grading of cancers. MiRNAs belong to the category of Cell-free nucleic. MiR-17 and miR-155 in body fluids can be used as biomarkers for the presence or absence of distant metastasis in breast cancer. MiR-182 had been proved to have potential value in molecular typing classification of breast cancer. ${ }^{23,24}$ Numerous studies have suggested that dysregulated miRNAs are associated with multidrug resistance in BC. Existing research revealed that miR-21 contributed to gemcitabine resistance by regulating its target gene, phosphatase and tensin homolog, in $\mathrm{BC}{ }^{25} \mathrm{Zhu}$ et al found miR-27b-3p to be associated with tamoxifen resistance in $\mathrm{BC} .{ }^{26}$ Moreover, Studies show that miR-451/452 was associated with the resistance of $\mathrm{BC}$ cells to Adriamycin. ${ }^{27,28}$ Recent data indicated that miR-137 directly targeted dual-specificity phosphatase 4 (DUSP4) to impact the chemoresistance 

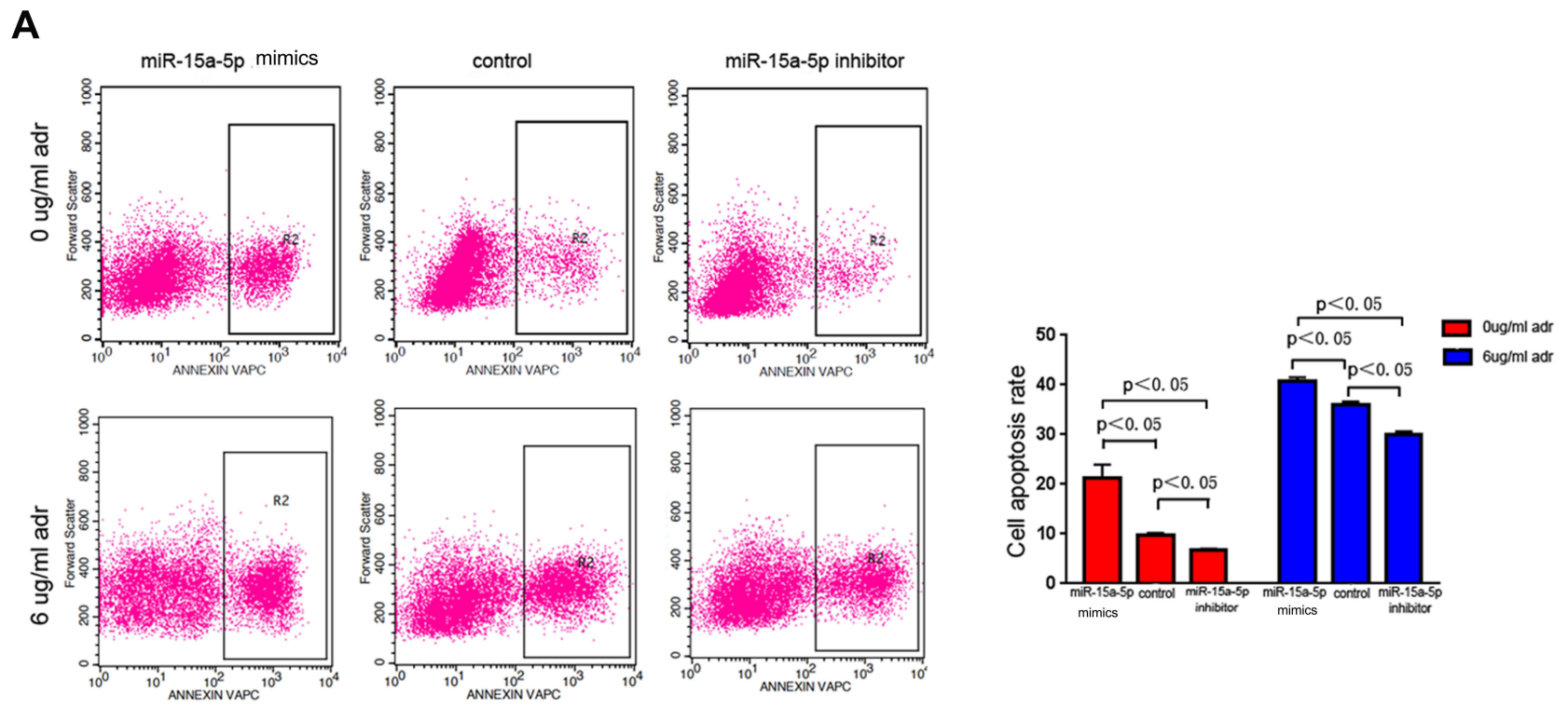

B
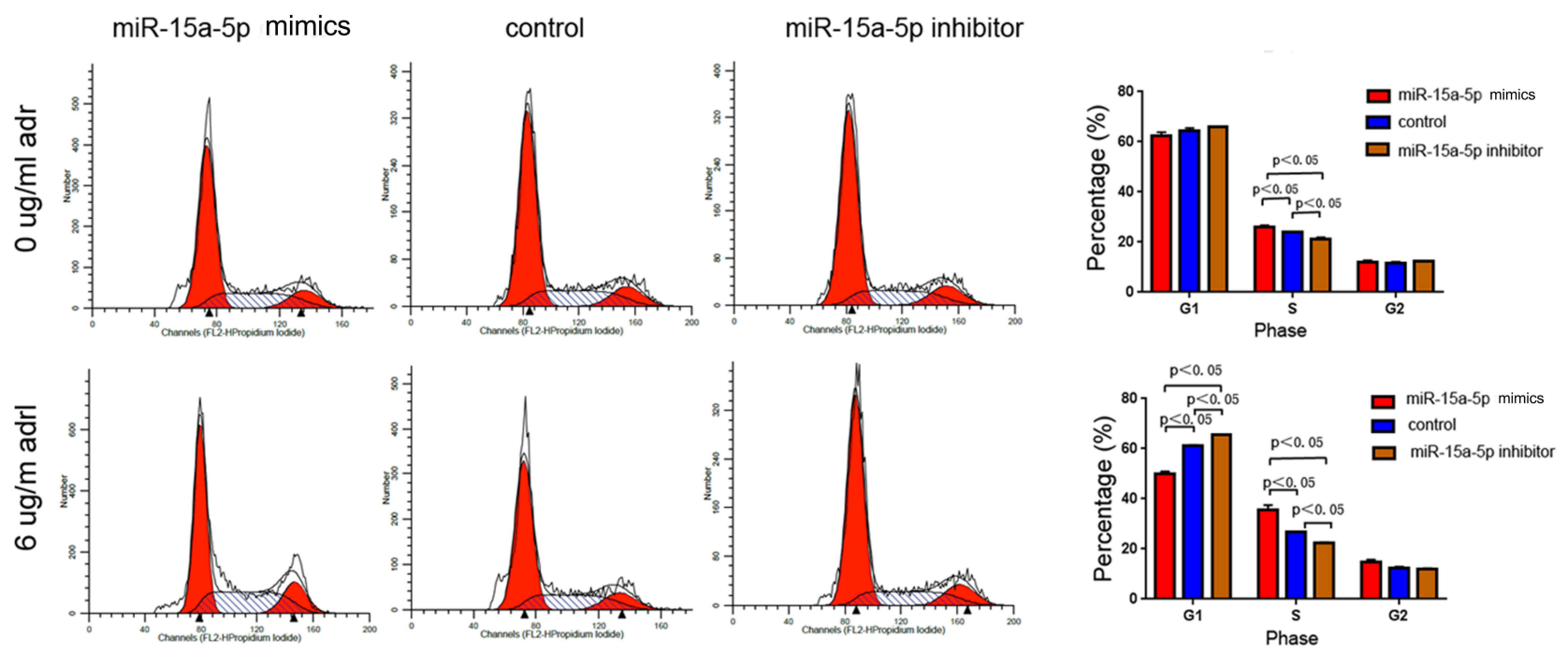

Figure 4 Apoptosis and the cell-cycle distribution of Michigan Cancer Foundation-7/Adriamycin (MCF-7/ADR) cells, assessed using flow cytometry. (A) Microribonucleic acid-15a-5p (MiR-I5a-5p) overexpression in MCF-7/ADR cells significantly induced apoptosis, particularly under Adriamycin pressure. Conversely, under the same conditions, low miR-15a-5p expression inhibited MCF-7/ADR cell apoptosis. (B) The rates of cells arrested in the synthesis phase were significantly elevated and reduced in the miR-15a-5p mimic and inhibitor groups, respectively, compared with the control group $(P<0.05)$.

of $\mathrm{BC}$ cells upon treatment with Adriamycin. ${ }^{29}$ Hong et al demonstrated that miR-7 markedly reversed paclitaxel/carboplatin resistance in $\mathrm{BC}$ by restoring the expression of multidrug resistance protein 1 (MRP1) and B-cell lymphoma 2 (BCL-2). ${ }^{30}$ Furthermore, research showed that the overexpression of miR-15a/miR-16 suppressed the target gene, B lymphoma Mo-MLV insertion region 1 (BMI1), to enhance BC cell sensitivity to Adriamycin, thereby suggesting new therapeutic targets that could help to improve the effects of chemotherapy in patients that experienced Adriamycin-resistant BC. ${ }^{31}$

\section{miR-15a-5p in Adriamycin-Resistant of Breast Cancer}

In this study, we assessed the function and molecular mechanisms of miR-15a-5p in Adriamycin-resistant BC. We first confirmed that miRNA-15a-5p was involved in $\mathrm{BC}$ resistance to Adriamycin, likely by regulating its 


\section{A}

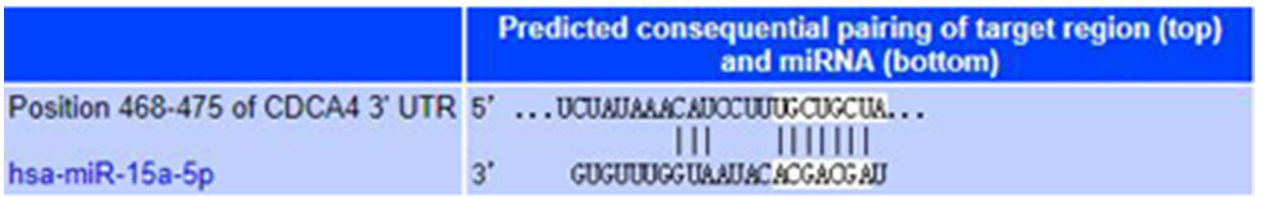

B

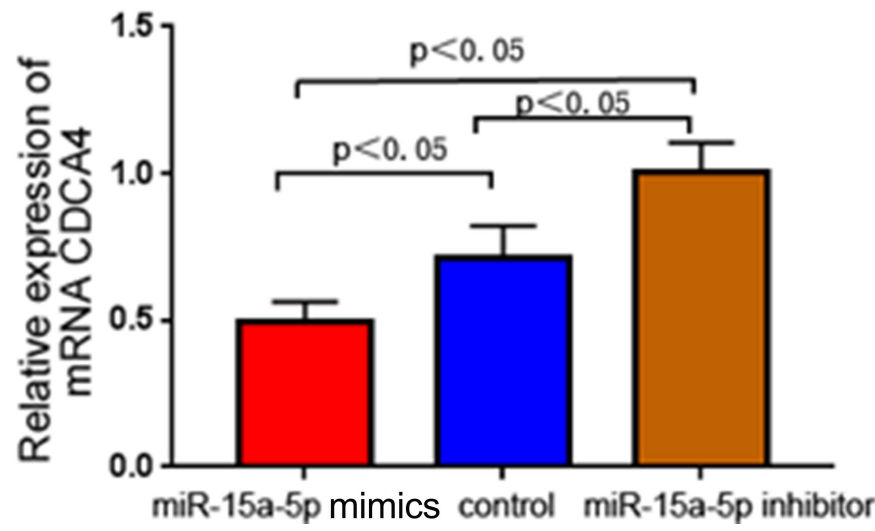

Figure 5 Microribonucleic acid-I5a-5p (MiR-15a-5p) directly targets cycle-associated protein 4. (A) The predicted target sequence of miR-I5a-5p was determined by the TargetScanHuman miRNA database. (B) Relative expression of mRNA cycle-associated protein 4 was evaluated by quantitative reverse transcription-polymerase chain reaction in control and transfected Adriamycin (ADR)-resistant breast cancer cells MCF-7 (MCF-7/ADR) $(P<0.05)$.

target gene, CDCA4. As indicated by conducting qRTPCR, miR-15a-5p was starkly downregulated in MCF-7/ ADR cells vs MCF-7 cells, suggesting that miR-15a-5p downregulation may contribute to Adriamycin-resistance in BC. Additionally, functional tests, such as CCK-8, transwell assays, and flow cytometry, were carried out to assess MCF-7/ADR cells. As shown above, miR-15a-5p upregulation resulted in a decreased IC50 for Adriamycin, as well as proliferation, invasion, and migration in $\mathrm{MCF}-7 /$ ADR cells. Furthermore, apoptosis in MCF-7/ADR cells was induced by Adriamycin administration. These findings indicated that miR-15a-5p overexpression could, at least partially, reverse Adriamycin resistance in $\mathrm{BC}$ and increase BC cell sensitivity to Adriamycin. Therefore, in the presence of Adriamycin, MCF-7/ADR cell viability was markedly reduced following miR-15a-5p upregulation. Strong evidence showed that miRNAs regulate gene expression by inducing degradation, inhibiting the transcription of their targeted genes, and via participation in cellular activity. Therefore, to better understand the potential mechanisms of miR-15a-5p, three online miRNA databases were used to predict its targeted genes. All three databases identified CDCA4 as a target gene of miR-15a$5 \mathrm{p}$. This is supported by results presented by Alderman et al, who also indicate CDCA4 as a direct target of miRNA-15a, based on the results of the luciferase reporter assay.

\section{The Relationship of miR-I5a-5p and CDCA4 in Adriamycin-Resistant of Breast Cancer}

Our previous study demonstrated that CDCA4 amounts were markedly increased in BC tissue compared with normal tissue specimens, with CDCA4 reflecting an association with MCF-7/ADR cell viability. Low CDCA4 amounts inhibited proliferation and induced apoptosis in MCF-7/ADR cells following Adriamycin administration. The above results indicated that the effects of low CDCA4 expression were consistent with those of miR-15a-5p overexpression in MCF-7/ADR cells. Furthermore, qRT-PCR showed that miR-15a-5p overexpression downregulated CDCA4 in MCF-7/ADR cells. In contrast, CDCA4 was upregulated after miR-15a-5p silencing. Therefore, miR$15 \mathrm{a}-5 \mathrm{p}$ expression and CDCA4 amounts were negatively 
correlated, suggesting that miR-15a-5p had likely been involved in the formation of Adriamycin resistance in BC by targeting CDCA4.

In the present work, miR-15a-5p overexpression affected the cell cycle in $\mathrm{BC}$ cells, with more cells being arrested in the S-phase DNA replication. It is known that Adriamycin induces cancer-cell apoptosis by impacting the process of DNA synthesis in the $\mathrm{S}$ phase. Accordingly, we hypothesized that miR-15a-5p increased BC cell sensitivity to Adriamycin, likely via cell-cycle regulation.

\section{The miR-I5a-5p -CDCA4-Nrf2 Axle in Adriamycin-Resistant of Breast Cancer}

Our previous study showed that CDCA4, a downstream gene of nuclear erythroid 2-related factor 2 (Nrf2) signaling, contributed to Adriamycin resistance in $\mathrm{BC}$ cells via the regulation of cell-cycle progression. Mounting evidence demonstrates that Nrf2 is closely related to drug resistance in multiple cancers. ${ }^{32,33}$ In this study, we provide preliminary confirmation that miR-15a-5p plays a critical role in $\mathrm{BC}$ resistance to Adriamycin by targeting CDCA4. Therefore, the potential function of miR-15a-5p in Nrf2-signaling in Adriamycin-resistant BC deserves further investigation to comprehensively explore the mechanism of this treatment approach's resistance to BC.

\section{Conclusion}

To sum up, our study demonstrated that miR-15a-5p totally or partially reversed Adriamycin resistance in $\mathrm{BC}$ by direct targeting of CDCA4 through cell-cycle regulation, which is the first report in the field. And the study not only refined the molecular regulatory networks of breast cancer but also provide a new strategy to promote chemotherapy progression.

\section{Data Sharing Statement}

The datasets used and/or analysed during the current study available from the corresponding author on reasonable request.

\section{Consent for Publication}

Consent for publication was obtained from every individual whose data are included in this manuscript.

\section{Funding}

The National Natural Science Foundation of China (grant no. 81260341), The Guangxi Natural Science Foundation Program (NO. 2017JJA10173).

\section{Disclosure}

The authors report no conflicts of interest in this work.

\section{References}

1. DeSantis CE, Ma J, Gaudet MM, et al. Breast cancer statistics, 2019. CA Cancer J Clin. 2019;69(6):438-451. doi:10.3322/caac.21583

2. Acunzo M, Romano G, Wernicke D, Croce CM. MicroRNA and cancer-a brief overview. Adv Biol Regul. 2015;57:1-9.

3. Croset M, Pantano F, Kan CWS, et al. miRNA-30 family members inhibit breast cancer invasion, osteomimicry, and bone destruction by directly targeting multiple bone metastasis-associated genes. Cancer Res. 2018;78(18):5259-5273. doi:10.1158/0008-5472.CAN-17-3058

4. Hong BS, Ryu HS, Kim N, et al. Tumor suppressor miRNA-204-5p regulates growth, metastasis, and immune microenvironment remodeling in breast cancer. Cancer Res. 2019;79(7):1520-1534.

5. Long X, Shi Y, Ye P, Guo J, Zhou Q, Tang Y. MicroRNA-99a suppresses breast cancer progression by targeting FGFR3. Front Oncol. 2019;9:1473. doi:10.3389/fonc.2019.01473

6. Crigna AT, Samec M, Koklesova L, et al. Cell-free nucleic acid patterns in disease prediction and monitoring-hype or hope? EPMA J. 2020;11(4):1-25. doi:10.1007/s13167-020-00226-x

7. Shen $\mathrm{H}$, Wang $\mathrm{D}$, Li L, et al. MiR-222 promotes drug-resistance of breast cancer cells to Adriamycin via modulation of PTEN/Akt/ FOXO1 pathway. Gene. 2017;596:110-118. doi:10.1016/j. gene.2016.10.016

8. Li D, Wang X, Yang M, Kan Q, Duan Z. miR3609 sensitizes breast cancer cells to Adriamycin by blocking the programmed death-ligand 1 immune checkpoint. Exp Cell Res. 2019;380(1):20-28. doi:10.1016/j.yexcr.2019.03.025

9. Zhang W, Jiang H, Chen Y, Ren F. Resveratrol chemosensitizes Adriamycin-resistant breast cancer cells by modulating miR-122-5p. J Cell Biochem. 2019;120(9):16283-16292. doi:10.1002/jcb.28910

10. Utaijaratrasmi $P$, Vaeteewoottacharn $K$, Tsunematsu $T$, et al. The microRNA-15a-PAI-2 axis in cholangiocarcinoma-associated fibroblasts promotes migration of cancer cells. Mol Cancer. 2018;17 (1):10. doi:10.1186/s12943-018-0760-x

11. Shi L, Jackstadt R, Siemens H, Li H, Kirchner T, Hermeking H. p53induced miR-15a/16-1 and AP4 form a double-negative feedback loop to regulate epithelial-mesenchymal transition and metastasis in colorectal cancer. Cancer Res. 2014;74(2):532-542. doi:10.1158/ 0008-5472.CAN-13-2203

12. Bandi N, Vassella E. miR-34a and miR-15a/16 are co-regulated in non-small cell lung cancer and control cell cycle progression in a synergistic and Rb-dependent manner. Mol Cancer. 2011;10:55. doi:10.1186/1476-4598-10-55

13. Chu J, Zhu Y, Liu Y, et al. E2F7 overexpression leads to tamoxifen resistance in breast cancer cells by competing with E2F1 at miR-15a/ 16 promoter. Oncotarget. 2015;6(31):31944-31957. doi:10.18632/ oncotarget.5128

14. Fesler A, Liu H, Ju J. Modified miR-15a has therapeutic potential for improving treatment of advanced stage colorectal cancer through inhibition of BCL2, BMI1, YAP1 and DCLK1. Oncotarget. 2018;9 (2):2367-2383.

15. Cheong JK, Gunaratnam L, Zang ZJ, et al. TRIP-Br2 promotes oncogenesis in nude mice and is frequently overexpressed in multiple human tumors. $J$ Transl Med. 2009;7:8. doi:10.1186/1479-5876-7-8

16. Tang DJ, Hu L, Xie D, et al. Oncogenic transformation by SEI-1 is associated with chromosomal instability. Cancer Res. 2005;65 (15):6504-6508. doi:10.1158/0008-5472.CAN-05-0351

17. Tategu M, Nakagawa H, Hayashi R, Yoshida K. Transcriptional co-factor CDCA4 participates in the regulation of JUN oncogene expression. Biochimie. 2008;90(10):1515-1522. doi:10.1016/j. biochi.2008.05.014 
18. Hayashi R, Goto Y, Ikeda R, Yokoyama KK, Yoshida K. CDCA4 is an $\mathrm{E} 2 \mathrm{~F}$ transcription factor family-induced nuclear factor that regulates E2F-dependent transcriptional activation and cell proliferation. $\mathrm{J}$ Biol Chem. 2006;281(47):35633-35648. doi:10.1074/jbc.M603800200

19. Pang S, Xu Y, Chen J, Li G, Huang J, Wu X. Knockdown of cell division cycle-associated protein 4 expression inhibits proliferation of triple negative breast cancer MDA-MB-231 cells in vitro and in vivo. Oncol Lett. 2019;17(5):4393-4400.

20. Xu Y, Wu X, Li F, Huang D, Zhu W. CDCA4, a downstream gene of the Nrf2 signaling pathway, regulates cell proliferation and apoptosis in the MCF7/ADM human breast cancer cell line. Mol Med Rep. 2018;17(1):1507-1512.

21. Alderman C, Sehlaoui A, Xiao Z, Yang Y. MicroRNA-15a inhibits the growth and invasiveness of malignant melanoma and directly targets on CDCA4 gene. Tumour Biol. 2016;37(10):13941-13950. doi: $10.1007 /$ s 13277-016-5271-z

22. Pan J, Miao D, Chen L. Germacrone reverses Adriamycin resistance in human chronic myelogenous leukemia K562/ADM cells by suppressing MDR1 gene/P-glycoprotein expression. Chem Biol Interact. 2018;288:32-37. doi:10.1016/j.cbi.2018.04.012

23. Stastny I, Zubor P, Kajo K, Kubatka P, Golubnitschaja O, Dankova Z. Aberrantly methylated cfDNA in body fluids as a promising diagnostic tool for early detection of breast cancer. Clin Breast Cancer. 2020;20(6):e711-e22. doi:10.1016/j.clbc.2020.05.009

24. Qian S, Golubnitschaja O, Zhan X. Chronic inflammation: key player and biomarker-set to predict and prevent cancer development and progression based on individualized patient profiles. EPMA J. 2019;10(4):365-381. doi:10.1007/s13167-019-00194-X

25. Wu ZH, Tao ZH, Zhang J, Li T, Hu XC. MiRNA-21 induces epithelial to mesenchymal transition and gemcitabine resistance via the PTEN/AKT pathway in breast cancer. Tumour Biol J Int Soc Oncodevelopmental Biol Med. 2015;37(6):7245-7254. doi:10.1007/ s13277-015-4604-7
26. Zhu J, Zou Z, Nie P, et al. Downregulation of microRNA-27b-3p enhances tamoxifen resistance in breast cancer by increasing NR5A2 and CREB1 expression. Cell Death Dis. 2016;7(11):e2454. doi:10.1038/cddis.2016.361

27. Kovalchuk O, Filkowski J, Meservy J, et al. Involvement of microRNA-451 in resistance of the $\mathrm{MCF}-7$ breast cancer cells to chemotherapeutic drug doxorubicin. Mol Cancer Ther. 2008;Jul (7):2152-2159. doi:10.1158/1535-7163.MCT-08-0021

28. Hu Q, Gong JP, Li J, Zhong SL, Tang J-H. Down-regulation of miRNA-452 is associated with adriamycin-resistance in breast cancer cells. Asian Pacific J Cancer Prev. 2014;15(13):5137-5142. doi:10.7314/APJCP.2014.15.13.5137

29. Du F, Yu L, Wu Y, et al. miR-137 alleviates doxorubicin resistance in breast cancer through inhibition of epithelial-mesenchymal transition by targeting DUSP4. Cell Death Dis. 2019;10(12):922. doi:10.1038/ s41419-019-2164-2

30. Hong T, Ding J, Li W. miR-7 reverses breast cancer resistance to chemotherapy by targeting MRP1 and BCL2. Onco Targets Ther. 2019;12:11097-11105. doi:10.2147/OTT.S213780

31. Patel N, Garikapati KR, Pandita RK, et al. miR-15a/miR-16 down-regulates BMI, impacting $\mathrm{Ub}-\mathrm{H} 2 \mathrm{~A}$ mediated DNA repair and breast cancer cell sensitivity to doxorubicin. Sci Rep. 2017;7 (1):4263. doi:10.1038/s41598-017-02800-2

32. Wang H, Liu K, Chi Z, et al. Interplay of MKP-1 and Nrf2 drives tumor growth and drug resistance in non-small cell lung cancer. Aging. 2019;11(23):11329-11346. doi:10.18632/aging.102531

33. Yen $\mathrm{CH}$, Hsiao HH. NRF2 is one of the players involved in bone marrow mediated drug resistance in multiple myeloma. Int J Mol Sci. 2018;19(11):3503. doi:10.3390/ijms19113503

\section{Publish your work in this journal}

Cancer Management and Research is an international, peer-reviewed open access journal focusing on cancer research and the optimal use of preventative and integrated treatment interventions to achieve improved outcomes, enhanced survival and quality of life for the cancer patient.
The manuscript management system is completely online and includes a very quick and fair peer-review system, which is all easy to use. Visit http://www.dovepress.com/testimonials.php to read real quotes from published authors. 